\title{
Reliability Guaranteed Energy Minimization On Mixed-Criticality Systems
}

\author{
Zheng Li ${ }^{\mathrm{a}}$, Chunhui Guo ${ }^{\mathrm{b}}$, Xiayu Hua ${ }^{\mathrm{b}}$, Shangping Ren ${ }^{\mathrm{b}}$ \\ ${ }^{a}$ Western Illinois University, Macomb, IL 61455, USA \\ ${ }^{b}$ Illinois Institute of Technology, Chicago, IL 60616, USA
}

\begin{abstract}
This paper studies the energy minimization problem in mixed-criticality systems that have stringent reliability and deadline constraints. We first analyze the resource demand of a mixed-criticality task set that has both reliability and deadline requirements. Based on the analysis, we present a heuristic task scheduling algorithm that minimizes system's energy consumption and at the same time also guarantees system's reliability and deadline constraints. Extensive experiments are conducted to evaluate and validate the performance of the proposed algorithm. The empirical results show that the algorithm further improves energy saving by up to $10 \%$ compared with the approaches proposed in our earlier work.
\end{abstract}

Keywords: Mixed-criticality, Real-time, Reliability, Energy

\section{Introduction}

For real-time embedded systems, to further reduce system cost, more and more tasks with different functionality and of different levels of criticality are integrated on the same hardware platform [10, 13]. Examples include recent unmanned aerial vehicles (UAV) which integrate the HI-criticality tasks such as flight-control tasks and LO-criticality tasks such as photo capturing tasks on the same platform [4]. Though the mixed-criticality design paradigm reduces system cost, tasks of different criticalities compete for the shared resource and cause system's timing behaviors become less predictable.

For a safety-critical system, high-criticality tasks are more crucial to the entire system than low-criticality tasks. To ensure that HI-criticality tasks always meet their deadlines, two worst case execution times are set for each HI-criticality task, i.e., worst case execution time by design and a more pessimistic one, worst case execution time by certification. When tasks' actual execution time is no more than their designed worst case execution time, the system is considered operating under the LOmode. However, if any task executes beyond this limit, the system changes to the HI-mode immediately to signal that a situation beyond designed behaviors has occurred and some actions need to take place. A mixed-criticality system is schedulable if the following two conditions are satisfied [12]: 1) both LOcriticality and HI-criticality tasks are guaranteed to meet their deadlines under the LO-mode; and 2) HI-criticality tasks are also guaranteed to meet their deadlines under the HI-mode. Determining whether a given mixed-criticality system is schedulable has been proven to be NP-hard [5] and different heuristic approaches are developed to addressing the schedulability issue.

Email addresses: z-1i2@wiu. edu (Zheng Li), cguo13@iit.edu (Chunhui Guo), xhua@it. edu (Xiayu Hua), ren@iit.edu (Shangping Ren)
In addition to guarantee task deadlines, power/energy efficiency and reliability issues are also critical for real-time embedded systems. As more and more transistors are integrated into a single chip, operation power/energy consumption of the chip has increased exponentially. Dynamic Voltage and Frequency Scaling (DVFS) technique, which dynamically lowers down the supply voltage and working frequency, is widely used for power/energy management. However, existing work [19. 28] has shown that transient fault rate increases when the supply voltage on the chip scales down. In other words, lowering down system's supply voltage potentially degrades the system's reliability. Hence, minimizing system's energy consumption without sacrificing the reliability requirement is another design challenge.

In this paper, we study how to schedule a mixed-criticality task set to minimize system's energy consumption under the following constraints:

1. schedulability constraint: both HI-criticality and LO-criticality tasks are guaranteed to meet their deadlines under LO-mode, and HI-criticality tasks are guaranteed to meet their deadlines under HI-mode;

2. reliability constraint: both HI-criticality and LO-criticality tasks are guaranteed to meet their reliability constraints under LO-mode, and HI-criticality tasks are guaranteed to meet their reliability constraints under HI-mode.

The main contributions of this paper are three-fold:

1. theoretically analyze the resource demand of mixed-criticality task set under both reliability and schedulability constraints;

2. develop a heuristic search based frequency assignment (HSFA) algorithm that decides the lowest task execution frequency which guarantees both deadline and reliability constraints; 
3. empirically evaluate and compare the energy saving performance of the HSFA algorithm with the state-of-the-art approaches in the literature.

The rest of the paper is organized as follows. We first discuss related work in Section 2 and then present the models and definitions the paper is based upon in Section 3. The research problem to be addressed in the paper is formally defined in Section 4. The theoretical foundations are established in Section 5 Based on the theoretical analysis, we present a heuristic search based frequency assignment algorithm in Section 6. Experimental results are discussed in Section 7 and finally we conclude our work in Section 8 .

\section{Related work}

The study of mixed-criticality task set scheduling issue started in the recent years. Baruah [7] first proposed to apply earliest deadline first (EDF) scheduling theory in mixedcriticality task set scheduling. To ensure the schedulability of a mixed-criticality task set, the earliest deadline first with virtual deadline (EDF-VD) scheduling algorithm was proposed [6]. The EDF-VD algorithm assigns HI-criticality tasks reduced deadlines to ensure that HI-criticality tasks are schedulable even if they overun their normal worst case execution time. Ekberg [12, 13] proposed a greedy approach by using the demandbound function analysis to determine a task set's schedulability under EDF algorithm. Ekberg's greedy algorithm has a significant improvement over the EDF-VD, but it has higher time complexity. However, both Baruah's EDD-VD and Ekberg's greedy algorithm take the approach of terminating all LO-criticality tasks if any instance of HI-criticality task overruns its normal worst case execution time, i.e., when system enters into the HI-mode.

To provide a guaranteed minimum level of service to LOcriticality tasks when system enters into the HI-mode, $\mathrm{Su}$ [22] considered using elastic task models [11] to increase

LO-criticality tasks' period and hence reduce their competition against HI-criticality tasks but allow LO-criticality tasks to execute when possible. By noticing that postponing HI-criticality tasks' execution can promote earlier execution of LO-criticality tasks, Park [20] developed a scheme called criticality based EDF which delays the execution of HI-criticality tasks as late as possible but without causing deadline violations.

Energy saving and reliability are two other major concerns for real-time embedded systems. For single criticality real-time systems, i.e. all tasks in the system have the same criticality, Zhu et al. [27] proposed a reliability-aware power management scheme which aims to minimize energy consumption while at the same time maintain system's reliability at the same level as if all tasks were executed with the highest processing frequency. Zhao et al. later improved the approach and developed a shared recovery technique that allows all tasks to share the same reserved recovery block, but only allows a single fault recovery during the entire task set execution [24]. Zhao et al. [25] further extended the work and developed a generalized shared recovery technique which removed single recovery constraint and allow multiple fault recoveries by reserving multiple recovery blocks.

Recently, the study of energy saving and reliability issues in the context of mixed-criticality task sets has drawn increased attention. With the objective to minimize system's energy consumption and at the same time guarantee task deadlines, Huang et al. [15] presented an approach by utilizing DVFS and EDFVD techniques [6] to solve the problem. To satisfy system's reliability requirements, Axer et al. [1] developed an approach to tolerating transient faults by duplicating high criticality tasks. Pathan et al. [21] also proposed a fixed-priority scheduling algorithm to tolerate transient faults in mixed-criticality systems.

As we can see, the aforementioned work treats reliability and energy consumption issues independently. However, system reliability and energy minimization are correlated when DVFS is used. In particular, reduced processing frequency reduces system energy consumption, but at the same time, reduced processing frequency also increases transient failure rate. Hence, they need to be addressed conjointly. In this paper, different with Huang et al.'s work [15] that reliability constraint is not taken into consideration, we are to address the problem about how to schedule mixed-criticality task sets to minimize system's energy consumption while at the same time satisfying both deadline and reliability constraints.

\section{Models and Definitions}

In this section, we introduce the models and definitions the research is based upon.

\subsection{Models}

\section{Processor Model}

The processor is DVFS enabled with a finite set of available frequencies, i.e. $F=\left\{f_{1}, \ldots, f_{q}\right\}$. The frequency values in $F$ are in a descending order with $f_{1}=f_{\max }$ and $f_{q}=f_{\min }$. These frequencies are normalized with respected to $f_{\max }$, i.e., $f_{\max }=1$.

\section{Task Model}

In this paper, we make the same assumptions as in [6, 13], i.e. there are two different criticality levels in a task set. In particular, for a given mixed-criticality task set $\Gamma=\left\{\tau_{1}, \tau_{2}, \ldots, \tau_{n}\right\}$, each task $\tau_{i}$ is defined by a quadruple as $\tau_{i}=\left(L_{i}, C_{i}, T_{i}, D_{i}\right)$, where

- $T_{i}$ is the task's period,

- $D_{i}$ is the task's relative deadline and we assume $D_{i} \leq T_{i}$,

- $L_{i} \in\{\mathrm{LO}, \mathrm{HI}\}$ is the task's criticality level,

- $C_{i}=\left\{C_{i}(\mathrm{LO}), C_{i}(\mathrm{HI})\right\}$ is task's worst-case execution time and $C_{i}(\chi)$ is the worst case execution time at criticality level $\chi$ under maximum processing frequency $f_{\max }$. If $\tau_{i}$ is a HI-criticality task, $C_{i}(\mathrm{LO}) \leq C_{i}(\mathrm{HI})$; while if $\tau_{i}$ is a LO-criticality task, $C_{i}(\mathrm{LO})=C_{i}(\mathrm{HI})$. 
The LO-criticality subset and the HI-criticality subset are denoted as $\Gamma_{L}$ and $\Gamma_{H}$, respectively.

\section{Transient Fault Model}

Although both permanent and transient faults may occur during task execution, transient faults are found more frequent than permanent faults [14, 19]. Hence, in this paper, we focus on transient faults. We adapt the same assumption as in the literature that the transient fault rate follows Poisson distribution with an average fault rate $\lambda[18,25,27]$. When a system is running under frequency $f_{i}$, the average transient fault rate is expressed as

$$
\lambda\left(f_{i}\right)=\hat{\lambda}_{0} 10^{-\hat{d} f_{i}}
$$

where $\hat{\lambda}_{0}=\lambda_{0} 10^{\frac{d}{1-f_{\min }}}, \hat{d}=\frac{d}{1-f_{\min }}$, and $\lambda_{0}$ is the average fault arrival rate when system running under the maximum frequency $f_{\max }$. The value $d(>0)$ is a system-dependent constant, which indicates the sensitivity of the system's fault arrival rate to system voltage and frequency scaling, the larger the $d$ value is, the more sensitive the fault arrival rate to voltage and frequency scaling.

\section{Fault Recovery Model}

Backward fault recovery [26] is used in our system to recover failed task instances. We assume fault detection is taken at the end of a task instance's execution. If a fault is detected, the failed task instance is re-executed at the maximum processing speed $\left(f_{\max }\right)$ and the re-execution must be completed within the same instance period. The time cost of fault detection is considered to be part of task's worst case execution time.

\section{System Execution Model}

The system has two execution modes, i.e. the LO-mode and the HI-mode. Initially, the system executes at the LOmode. In the LO-mode, each task $\tau_{i}$ can execute up to $\frac{C_{i}(\mathrm{LO})}{f\left(\tau_{i}\right)}$ time units within each period under frequency $f\left(\tau_{i}\right)$. When any task $\tau_{i}$ executes beyond $\frac{C_{i}(\mathrm{LO})}{f\left(\tau_{i}\right)}$ time within its period, the system switches to the HI-mode immediately. In the HI-mode, all LO-criticality tasks are removed from further execution and every HI-criticality task $\tau_{i}$ 's maximum execution time changes to $\frac{C_{i}(\mathrm{HI})}{f^{\prime}\left(\tau_{i}\right)}$ if $f^{\prime}\left(\tau_{i}\right)$ is task $\tau_{i}$ 's processing frequency under the HI-mode.

It is worth pointing out that fault recovery time is not counted as task execution time. More specifically, when a task $\tau_{i}$ executes under frequency $f\left(\tau_{i}\right)$ for $t_{1}$ time units but fails, and it takes additional $t_{2}$ time to do the recovery, if $t_{1}+t_{2} \geq \frac{C_{i}(\mathrm{LO})}{f\left(\tau_{i}\right)}$ but $t_{1}<\frac{C_{i}(\mathrm{LO})}{f\left(\tau_{i}\right)}$, the system still operates under the LO-mode and does not change to the HI-mode. In other words, system mode change is triggered only when $t_{1} \geq \frac{C_{i}(\mathrm{LO})}{f\left(\tau_{i}\right)}$.

\section{Task-Instance-Level Reliability Model}

Task-instance-level reliability is defined as the probability of completing a task instance without incurring errors caused by transient faults [26]. It is calculated as

$$
r_{i}\left(f\left(\tau_{i}\right)\right)=e^{-\lambda\left(f\left(\tau_{i}\right)\right) \cdot \frac{C_{i}}{f\left(\tau_{i}\right)}}
$$

where $C_{i}$ and $f\left(\tau_{i}\right)$ are the task instance's execution time and system's working frequency, respectively.

\section{Task-Level Reliability Model}

Task-level reliability is defined as the probability of completing all instances of task $\tau_{i}$ successfully within a hyperperiod of a given task set. It is denoted as $R_{i}$. If $\tau_{i}$ has $k_{i}$ instances within a hyperperiod $H$, all these $k_{i}$ instances are executed under $f\left(\tau_{i}\right)$, and at most $\delta_{i}$ among them are allowed to be recovered, then the reliability $\left(R_{i}\right)$ of task $\tau_{i}$ can be calculated as [24]

$$
\begin{aligned}
& R_{i}\left(f\left(\tau_{i}\right), \delta_{i}, k_{i}\right)= \\
& \left(r_{i}\left(f\left(\tau_{i}\right)\right)\right)^{k}+\sum_{j=1}^{\delta_{i}}\left(\begin{array}{l}
k_{i} \\
j
\end{array}\right)\left(1-r_{i}\left(f\left(\tau_{i}\right)\right)\right)^{j}\left(r_{i}\left(f\left(\tau_{i}\right)\right)\right)^{k_{i}-j} .
\end{aligned}
$$

\section{Energy Model}

We use the same power/energy model given in [18, 25, 27]. In particular, under the processing frequency $f\left(\tau_{i}\right)$, system's power consumption is represented as

$$
P\left(f\left(\tau_{i}\right)\right)=P_{s}+h P_{a}=P_{s}+h\left(P_{\text {ind }}+C_{\text {ef }}\left(f\left(\tau_{i}\right)\right)^{\theta}\right),
$$

where $P_{s}$ is the static power used to maintain the system in a standby state. The static power cannot be saved unless the system is turned off. $P_{a}$ is the active power used when the system is in the working state. $P_{a}$ has two components, frequency independent power $\left(P_{\text {ind }}\right)$, such as the power used for memory and I/O operations, and frequency dependent power $\left(C_{\text {ef }} f^{\theta}\right)$. Parameters $C_{\text {ef }}$ and $\theta$ are system dependent constants and $\theta \geq 2$ [2,9]. Boolean parameter $h=1$ indicates the system is in the working state, and $h=0$ indicates the system is in the standby state.

We assume the system is always on and hence focus only on active power saving. As the energy consumption due to voltage and frequency scaling is independent of $P_{s}$, without loss of generality, we set $P_{s}=0$. In addition, we also make the same assumption as in [3] that task execution time is linearly related to working frequency. Therefore, under the working frequency $f\left(\tau_{i}\right)$, task $\tau_{i}$, s execution time is $\frac{C_{i}}{f\left(\tau_{i}\right)}$ and the energy consumption within one period can be represented as

$$
\begin{aligned}
E\left(f\left(\tau_{i}\right), c_{i}\right) & =\left(P_{\text {ind }}+C_{\text {ef }} f\left(\tau_{i}\right)^{\theta}\right) \frac{c_{i}}{f\left(\tau_{i}\right)} \\
& =P_{\text {ind }} \frac{c_{i}}{f\left(\tau_{i}\right)}+C_{\text {ef }} C_{i} f\left(\tau_{i}\right)^{\theta-1} .
\end{aligned}
$$

From Equation (5), it is clear that scaling down the processing frequency reduces frequency dependent energy, i.e. $C_{\mathrm{ef}} C_{i} f\left(\tau_{i}\right)^{\theta-1}$. Hence, there is a balanced frequency, i.e. the energy-efficient frequency $\left(f_{\mathrm{ee}}\right)$ - further scaling down the processing frequency below $f_{\text {ee }}$ will increase total energy consumption. Early study [27] concludes that

$$
f_{\mathrm{ee}}=\sqrt[\theta]{\frac{P_{\text {ind }}}{C_{\mathrm{ef}}(\theta-1)}} .
$$

To simplify the discussion, we further assume $f_{\min } \geq f_{\text {ee }}$. 


\subsection{Definitions}

We introduce the following definitions which will be used in the paper.

Task $\tau_{i}$ LO-mode utilization $u_{L}\left(\tau_{i}\right): u_{L}\left(\tau_{i}\right)=\frac{C_{i}(\mathrm{LO})}{T_{i}}$.

Task $\tau_{i}$ HI-mode utilization $u_{H}\left(\tau_{i}\right): u_{H}\left(\tau_{i}\right)=\frac{C_{i}(\mathrm{HO})}{T_{i}}$.

LO-criticality task set $\chi$-mode utilization $U_{\chi}\left(\Gamma_{L}\right)$ : $U_{\chi}\left(\Gamma_{L}\right)=\sum_{\tau_{i} \in \Gamma_{L}} u_{\chi}\left(\tau_{i}\right)$, where $\chi \in\{$ HI, LO $\}$.

HI-criticality task set $\chi$-mode utilization $U_{\chi}\left(\Gamma_{H}\right)$ : $U_{\chi}\left(\Gamma_{H}\right)=\sum_{\tau_{i} \in \Gamma_{H}} u_{\chi}\left(\tau_{i}\right)$, where $\chi \in\{\mathrm{HI}, \mathrm{LO}\}$.

\section{Problem Formulation}

Based on the defined models, we define the problem this paper is to address as below:

Problem 1. Given a DVFS enabled processor with $q$ different processing frequencies, i.e. $F=\left\{f_{1}, \cdots, f_{q}\right\}$, where $f_{1}=$ $f_{\max }, f_{q}=f_{\min }$ and $f_{i}>f_{j}$ if $i<j$, and a mixed-criticality task set $\Gamma=\left\{\Gamma_{L}, \Gamma_{H}\right\}$, develop a scheduling algorithm that minimizes system's energy consumption under the following two constraints:

1. schedulability constraint: HI-criticality and LO-criticality tasks are both guaranteed to meet their deadlines under the LO-mode, and HI-criticality tasks are guaranteed to meet their deadlines under the HI-mode;

2. reliability constraint: $H I$-criticality and LO-criticality tasks are both guaranteed to meet their reliability constraint under the LO-mode, and HI-criticality tasks are guaranteed to meet their reliability constraints under the HI-mode.

The EDF-VD algorithm [6, 12, 23] is a widely used scheduling algorithm for mixed-criticality task sets. Under the EDFVD algorithm, when system operates under the LO-mode, tasks are scheduled using EDF algorithm with reduced deadlines which is also called virtual deadlines (VD). However, when the system changes to the HI-mode, all LO-criticality tasks are removed from further execution, and each HI-criticality task's deadline is reset to its original deadline to guarantee that even in the overrun situation tasks can still meet their deadlines. In this paper, we assume that the EDF-VD algorithm is used to schedule the mixed-criticality task sets. Due to the improbability of system switching to the HI-mode [15], for deadline guarantee and energy saving purposes, we run tasks at $f_{\max }$ under the HI-mode and use lower frequencies when the system executes under the LO-mode.

However, in order to actually implement the above task execution strategy, we have to answer under what frequency $f\left(\tau_{i}\right)$ and with what virtual deadline $\mathrm{VD}_{i}$ each task $\tau_{i}$ should be executed when the system is in the LO-mode so that the energy consumption is minimized and system schedulability and reliability constraints are guaranteed. We address the question in three steps: first, determine whether the given task set working frequency $f\left(\tau_{i}\right)$ and virtual deadline $\mathrm{VD}_{i}$ satisfy system schedulability and reliability constraints; second, decide tasks' virtual deadline $\mathrm{VD}_{i}$ under given tasks' working frequency $f\left(\tau_{i}\right)$ that guarantees system schedulability and reliability constraints; and third, determine tasks' best working frequency $f\left(\tau_{i}\right)$ that minimizes energy consumption without violating system's schedulability and reliability constraints.

\section{Theoretical Foundations}

In this section, we establish the theoretical foundation that determines whether a mixed-criticality system's reliability and schedulability constraints are satisfied when each task $\tau_{i}$ 's processing frequency $f\left(\tau_{i}\right) \leq f_{\max }$ and its virtual deadline $\mathrm{VD}_{i} \leq$ $D_{i}$ are given.

\subsection{Satisfying Reliability Constraint}

According to the reliability model, i.e. Equation (3), scaling down task's working frequency increases transient fault rate and hence more recoveries are required to meet the reliability constraint. For a periodic task $\tau_{i}$, if the task-level reliability constraint is $R_{i}^{c}$ and the processing frequency under LO-mode is $f\left(\tau_{i}\right)$, according to Equation (3), the number of recoveries $\left(\delta_{i}^{L}\right)$ needed under the LO-mode to guarantee the system reliability constraint must satisfy the following Inequation:

$$
r_{i}\left(f\left(\tau_{i}\right)\right)^{k}+\sum_{j=1}^{\delta_{i}^{L}}\left(\begin{array}{l}
k_{i} \\
j
\end{array}\right)\left(1-r_{i}\left(f\left(\tau_{i}\right)\right)\right)^{j} r_{i}\left(f\left(\tau_{i}\right)\right)^{k_{i}-j} \geq R_{i}^{c} .
$$

Where,

$$
r_{i}\left(f\left(\tau_{i}\right)\right)=e^{-\lambda\left(f\left(\tau_{i}\right)\right) \cdot \frac{C_{i}(L O)}{f\left(\tau_{i}\right)}} .
$$

The value of $\delta_{i}^{L}$ can be obtained by solving Inequation (7). Similarly, the number of recoveries $\left(\delta_{i}^{H}\right)$ needed under HI-mode can be obtained by replacing $C_{i}(\mathrm{LO})$ with $C_{i}(\mathrm{HI})$ and $f\left(\tau_{i}\right)$ with $f_{\max }$ in the Inequation (7).

The reliability constraint of task $\tau_{i}$ is satisfied if at least $\delta_{i}^{L}$ and $\delta_{i}^{H}$ number of task instances are allowed to be recovered under the LO-mode and the HI-mode, respectively. The next question is, with the amount of recoveries, whether the schedulability constraint can also be satisfied?

\subsection{Satisfying Schedulability Constraint}

Based on the resource demand and supply analysis, a mixedcriticality task set is schedulable if the resource demand is no larger than the resource supply when system operates under both the LO-mode and HI-mode. In particularly, as illustrated in Figure 1, if the total execution duration is $x$ and system changes from the LO-mode to the HI-mode at time $x-y$, then the task set is schedulable if [23]

$$
\forall x, y \in[0, H] \wedge x \geq y: \sum_{\tau_{i} \in \Gamma} d b f_{i}^{\mathrm{LO}}(x-y) \leq x-y,
$$

and

$$
\forall x, y \in[0, H] \wedge x \geq y: \sum_{\tau_{i} \in \Gamma_{H}} d b f_{i}^{\mathrm{HI}}(x, y) \leq y .
$$




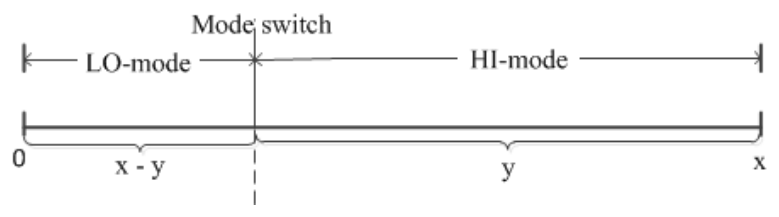

Figure 1: Illustration of mixed-criticality task execution

Among which, $d b f_{i}^{\mathrm{LO}}(x-y)$ is the resource demand of the task $\tau_{i}$ within the LO-mode execution duration $x-y$ and $d b f_{i}^{\mathrm{HI}}(x, y)$ is the resource demand within the HI-mode duration $y$ among the total execution duration $x$.

In the following, we discuss how to derive task's LO-mode and HI-mode resource demand to meet the schedulability constraint.

\subsubsection{LO-mode Resource Demand Analysis}

By definition, when a mixed-criticality system operates under the LO-mode, both LO-criticality and HI-criticality tasks should be guaranteed to meet their deadlines. In addition, to satisfy the reliability constraint, at least $\delta_{i}^{L}$ recoveries are required for each task $\tau_{i}$ within a given task set's hyperperiod. Therefore, to execute a mixed-criticality task set that has the reliability constraint, both the resource demand for task execution and fault recovery need to be taken into consideration.

Before providing task's resource demand under LO-mode, we first state a lemma [16] based on which to calculate the resource demand for task recoveries as follows:

Lemma 1. Given a task set $\Gamma=\left\{\tau_{1}, \ldots, \tau_{n}\right\}$ and their maximal allowed number of recoveries $\left\{\delta_{1}, \ldots, \delta_{n}\right\}$, the task set is schedulable if and only if all the deadlines are satisfied under the worst case scenario, i.e., when the first $\delta_{i}$ instances of all task $\tau_{i} \in \Gamma$ need to be recovered [16].

Based on Lemma1, we obtain the task set resource demand when the system operates under the LO-mode. It is given by Theorem 1

Theorem 1. For a given periodic task $\tau_{i}=\left(L_{i}, C_{i}, T_{i}, D_{i}\right)$, when system operates under the LO-mode, the task's working frequency is $f\left(\tau_{i}\right)$, the recovery allowance within one hyperperiod $H$ is $\delta_{i}^{L}$ and the virtual deadline is set as $V D_{i}$, then the resource demand of task $\tau_{i}$ within LO-mode duration $x-y(x \geq$ y) can be calculated as

$d b f_{i}^{L O}(x-y)=\left\{\begin{array}{l}N_{S} \cdot\left(1+\frac{f_{\max }}{f\left(\tau_{i}\right)}\right) \cdot C_{i}(L O), \quad \text { if } x-y \leq \delta_{i}^{L} \cdot T_{i} ; \\ \left(\delta_{i}^{L}+\left(\delta_{i}^{L}+N_{L}\right) \cdot \frac{f_{\max }}{f\left(\tau_{i}\right)}\right) \cdot C_{i}(L O), \text { otherwise. }\end{array}\right.$

where

$$
N_{S}=\left\lfloor\frac{x-y-V D_{i}}{T_{i}}\right\rfloor+1
$$

and

$$
N_{L}=\left\lfloor\frac{x-y-\delta_{i}^{L} \cdot T_{i}-V D_{i}}{T_{i}}\right\rfloor+1 .
$$

Proof. According to Lemma 1, the case that all the first $\delta_{i}^{L}$ instances of each task $\tau_{i} \in \Gamma$ needs to be recovered has the highest resource demand and we focus on the resource demand analysis under this case.

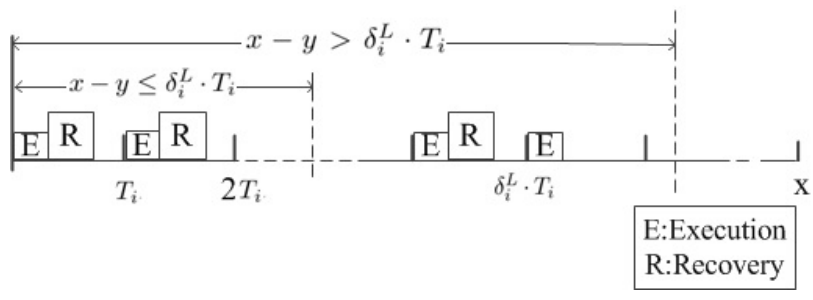

Figure 2: Illustration of LO-mode task execution and recovery

When $x-y \leq \delta_{i}^{L} \cdot T_{i}$, as illustrated in Figure 2, each instance needs one recovery running under $f_{\max }$ which takes $C_{i}(\mathrm{LO})$ time units. In addition, finishing each instance at frequency $f\left(\tau_{i}\right)$ also costs $\frac{f_{\max }}{f\left(\tau_{i}\right)} \cdot C_{i}(\mathrm{LO})$ time units. Sum them together, each instance of task $\tau_{i}$ demands $\left(1+\frac{f_{\max }}{f\left(\tau_{i}\right)}\right) \cdot C_{i}(\mathrm{LO})$ time units. For a given LO-mode duration $x-y$, at most $\left\lfloor\frac{x-y-\mathrm{VD}_{i}}{T_{i}}\right\rfloor+1$ task instances must be finished within the LOmode duration $x-y$. Therefore, if $x-y \leq \delta_{i}^{L} \cdot T_{i}$, the resource demand of task $\tau_{i}$ is at most

$$
\left(\left\lfloor\frac{x-y-\mathrm{VD}_{i}}{T_{i}}\right\rfloor+1\right) \cdot\left(1+\frac{f_{\max }}{f\left(\tau_{i}\right)}\right) \cdot C_{i}(\mathrm{LO}) .
$$

When $x-y>\delta_{i}^{L} \cdot T_{i}$, each of the first $\delta_{i}^{L}$ instances needs to recover once and hence the total resource demand of the first $\delta_{i}^{L}$ instances is

$$
\delta_{i}^{L} \cdot\left(1+\frac{f_{\max }}{f\left(\tau_{i}\right)}\right) \cdot C_{i}(\mathrm{LO}) .
$$

Since total $\left\lfloor\frac{x-y-\mathrm{VD}_{i}}{T_{i}}\right\rfloor+1$ task instances must finish within the LO-mode duration $x-y$, each of the remaining $\left\lfloor\frac{x-y-\mathrm{VD}_{i}}{T_{i}}\right\rfloor+$ $1-\delta_{i}^{L}$ instances only need to execute once without recovery. Therefore, the resource demand of these remaining instances is

$$
\left(\left\lfloor\frac{x-y-\delta_{i}^{L} \cdot T_{i}-\mathrm{VD}_{i}}{T_{i}}\right\rfloor+1\right) \cdot \frac{f_{\max }}{f\left(\tau_{i}\right)} \cdot C_{i}(\mathrm{LO}) .
$$

Let $N_{L}=\left\lfloor\frac{x-y-\delta_{i}^{L} \cdot T_{i}-\mathrm{VD}_{i}}{T_{i}}\right\rfloor+1$, then the total resource demand can be represented as

$$
\left(\delta_{i}^{L}+\left(\delta_{i}^{L}+N_{L}\right) \cdot \frac{f_{\max }}{f\left(\tau_{i}\right)}\right) \cdot C_{i}(\mathrm{LO}) .
$$

\subsubsection{HI-mode Resource Demand Analysis}

When system changes to the HI-mode, all the LO-criticality tasks are removed from further execution and all the 
HI-criticality tasks run under $f_{\max }$. Therefore, the resource demand comes only from HI-criticality tasks. Within the HI-mode execution duration of $y$, there are total $\left\lfloor\frac{y-D_{i}}{T_{i}}\right\rfloor$ task instances that are yet to be started and completed.

In addition to the yet to be started task instances, it is possible that at the mode switch point, there is a carry-over task instance of which part of its execution is completed in the LOmode under a scaled down frequency $f\left(\tau_{i}\right)<f_{\max }$ and it may already encounter a transient fault. The scenario is depicted in Figure 3 .

Initially, fault detection is only taken at the end of each task instance's execution, which means the fault of this carry-over task instance can only be detected after its remaining part is finished under the HI-mode. Resource would be wasted if a failed task is further executed. To avoid such resource waste, for carry-over task instances, in addition to taking fault detection at its end, we also assume the execution correctness of carry-over tasks is checked at the mode switch point. If a task instance is detected failed, we re-execute the task instance from the beginning in the HI-mode; otherwise, continue to finish its remaining part.

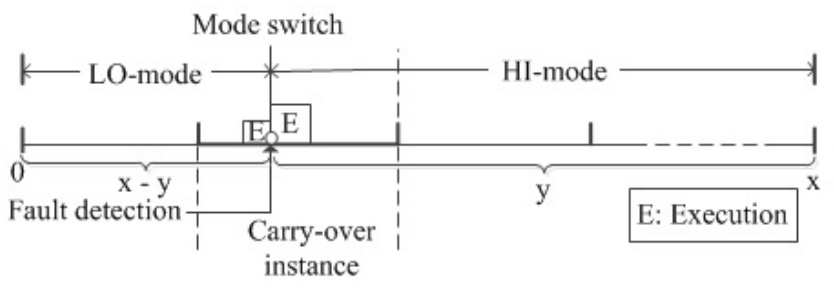

Figure 3: Illusration of carry-over task instance

Hence, in the worst-case scenario, all carry-over task instances need to be re-executed from the beginning and hence the resource demand of a HI-criticality task $\tau_{i}$ with the HI-mode duration $y$ is

$$
\left(\left\lfloor\frac{y-D_{i}}{T_{i}}\right\rfloor+1\right) \cdot C_{i}(\mathrm{HI})
$$

and the total resource demand of all HI-criticality tasks within the HI-mode execution duration of $y$ is

$$
\sum_{\tau_{i} \in \Gamma_{H}}\left(\left\lfloor\frac{y-D_{i}}{T_{i}}\right\rfloor+1\right) \cdot C_{i}(\mathrm{HI})
$$

However, using (13) to calculate the HI-mode resource demand is too pessimistic as it assumes every HI-criticality task has a carry-over instance which may not be the case. For instance, if the mode switch happens after a task instance's virtual deadline $\mathrm{VD}_{i}$ but before its deadline $D_{i}$, i.e. $(x-y)$ $\bmod T_{i}>\mathrm{VD}_{i}$, according to virtual deadline definition, this task does not have a carry-over instance.

The following theorem provides more accurate calculation of task resource demand under the HI-mode.

Theorem 2. For a given periodic task $\tau_{i}=\left(L_{i}, C_{i}, T_{i}, D_{i}\right)$, the virtual deadline is $V D_{i}$, the working frequency and recovery allowance under HI-mode are $f_{\max }$ and $\delta_{i}^{H}$ within one hyperperiod $H$, respectively, then the resource demand of task $\tau_{i}$ within HI-mode execution duration y among the total execution duration $x$ can be represented as

$$
d b f_{i}^{H I}(x, y)=\left(n_{i}(x, y)+\min \left\{n_{i}(x, y), \delta_{i}^{H}\right\}\right) \cdot C_{i}(H I),
$$

where

$$
n_{i}(x, y)=\left\{\begin{array}{l}
\max \left\{\left\lfloor\frac{y-D_{i}+\varepsilon_{i}}{T_{i}}\right\rfloor, 0\right\}, \text { if } \varepsilon_{i}>V D_{i} \\
\left\lfloor\frac{y-D_{i}+\varepsilon_{i}}{T_{i}}\right\rfloor+1, \text { if } \varepsilon_{i} \leq V D_{i} .
\end{array}\right.
$$

and $\varepsilon_{i}=(x-y) \bmod T_{i}$.

Proof. If $\varepsilon_{i}>\mathrm{VD}_{i}$, at the mode switch point, no carry-over task instance exists and all the released task $\tau_{i}$ instances are finished, and the next task instance will be released at $T_{i}-$ $\varepsilon_{i}$ time units after the mode switch point. Hence, within the HI-mode duration $y$, the number of task instances that must be finished is

$$
n_{i}(x, y)=\max \left\{\left\lfloor\frac{y-\left(T_{i}-\varepsilon_{i}\right)-D_{i}}{T_{i}}\right\rfloor+1,0\right\},
$$

which can be re-written as

$$
n_{i}(x, y)=\max \left\{\left\lfloor\frac{y-D_{i}+\varepsilon_{i}}{T_{i}}\right\rfloor, 0\right\} .
$$

When $\varepsilon_{i} \leq \mathrm{VD}_{i}$, task $\tau_{i}$ may have a carry-over instance. Under this scenario, there are three possible cases:

Case (1) $y<D_{i}-\varepsilon_{i}$ : since under the HI-mode, deadline of a HI-criticality task is $D_{i}, y<D_{i}-\varepsilon_{i}$ means this carry-over instance does not reach its deadline, hence, $n_{i}(x, y)=0$;

Case (2) $D_{i}-\varepsilon_{i} \leq y \leq T_{i}-\varepsilon_{i}$ : the carry-over instance has reached its deadline but no new task instance is released, hence, we only have the carry-over task instance to be finished. Therefore, $n_{i}(x, y)=1$;

Case (3) $y>T_{i}-\varepsilon_{i}$ : the carry-over instance has reached its deadline and new released task instances also need to be finished. In this case,

$$
\begin{aligned}
n_{i}(x, y) & =1+\left\lfloor\frac{y-\left(T_{i}-\varepsilon_{i}\right)-D_{i}}{T_{i}}\right\rfloor+1 \\
& =\left\lfloor\frac{y-D_{i}+\varepsilon_{i}}{T_{i}}\right\rfloor+1 .
\end{aligned}
$$

In fact, Equation 16 generalizes all the aforementioned three cases.

To satisfy the reliability constraint, each task $\tau_{i}$ is allowed $\delta_{i}^{H}$ number of instances to be recovered within one hyperperiod. According to Lemma 1 , the worst case from resource demand perspective is when the first $\delta_{i}^{H}$ instances of each task $\tau_{i}$ need to be recovered. In addition, under the HI-mode, task recovery under $f_{\max }$ may take up to $C_{i}(\mathrm{HI})$ time. Hence, the resource demand of task $\tau_{i}$ under the HI-mode for the duration of $y$ is

$$
\left(n_{i}(x, y)+\min \left\{n_{i}(x, y), \delta_{i}^{H}\right\}\right) \cdot C_{i}(\mathrm{HI}) .
$$


Based on resource demands under LO-mode, i.e. Equation (10) and HI-mode, i.e. Equation (14), we have the following lemma:

Lemma 2. For a given periodic task $\tau_{i}$ with period as $T_{i}$, when system operates under the LO-mode, the task's working frequency is $f\left(\tau_{i}\right)$ and the recovery allowance within one hyperperiod is $\delta_{i}^{L}$, the task's LO-mode resource demand will be monotonically nondecreasing when decreasing the assigned virtual deadline $V D_{i}$.

Proof. Task's LO-mode resource demand can be calculated by Equation (10), which can be re-written as

$$
d b f_{i}^{\mathrm{LO}}(x-y)=N_{S} \cdot \frac{f_{\max }}{f\left(\tau_{i}\right)} \cdot C_{i}(\mathrm{LO})+\min \left\{N_{S}, \delta_{i}^{L}\right\} \cdot C_{i}(\mathrm{LO}),
$$

where $x$ is the total execution duration and $x-y$ is the LOmode execution duration. It is not hard to find that, under given $x-y>0$, reducing $\mathrm{VD}_{i}$ may increase but never decrease the value of $N_{S}=\left\lfloor\frac{x-y-\mathrm{VD}_{i}}{T_{i}}\right\rfloor$, and hence $d b f_{i}^{\mathrm{LO}}(x-y)$ will never be decreased.

Lemma 3. For a given periodic task $\tau_{i}$ with period as $T_{i}$ and deadline as $D_{i}$, the recovery allowance within one hyper-period under HI-mode is $\delta_{i}^{H}$, then the task's HI-mode resource demand will be monotonically nonincreasing when decreasing the assigned virtual deadline $V D_{i}$.

Proof. The HI-mode resource demand can be calculated using Equation (14), i.e.

$$
d b f_{i}^{\mathrm{HI}}(x, y)=\left(n_{i}(x, y)+\min \left\{n_{i}(x, y), \delta_{i}^{H}\right\}\right) \cdot C_{i}(\mathrm{HI}),
$$

where $x$ is the total execution duration and $y$ is the HI-mode execution duration. $n_{i}(x, y)$ can be calculated based on Equation (15). From Equation (15), it is clearly that $n_{i}(x, y)$ may be decreased but never be increased by reducing the value of $\mathrm{VD}_{i}$. Therefore, $d b f_{i}^{\mathrm{HI}}(x, y)$ is monotonically nonincreasing when decreasing $\mathrm{VD}_{i}$.

\section{Heuristic Search Based Approach to Minimizing Energy Consumption}

In Section 5, Theorem 1 and Theorem 2 are established to check whether a mixed-criticality task set with the reliability constraint is schedulable under given task's virtual deadline and processing frequency assignments. In this section, we first present a virtual deadline assignment strategy and based on the virtual deadline assignment, we then give a processing frequency to task assignment algorithm that satisfies both schedulability and reliability constraints and also results in the least energy consumption.

\subsection{Virtual Deadline Assignment}

Given a task $\tau_{i}$ 's virtual deadline as $\mathrm{VD}_{i}$, Inequation (8) and (9) together are able to check whether the task set is schedulable. The question is how to decide the virtual deadline $\mathrm{VD}_{i}$ for each task that satisfies the Equation (8) and (9).
A closer look reveals that satisfying Inequation (8) and (9) is equivalent to satisfy the following Inequation (17) and (18) for $\forall x \in[0, H]$, respectively, i.e.

$$
\sum_{\tau_{i} \in \Gamma} d b f_{i}^{\mathrm{LO}}(x) \leq x
$$

and

$$
\forall y \in[0, x]: \sum_{\tau_{i} \in \Gamma_{H}} d b f_{i}^{\mathrm{HI}}(x, y) \leq y
$$

Furthermore, based on the Lemma 3, decreasing $\mathrm{VD}_{i}$ potentially decreases task's resource demand under the HI-mode and hence Inequation (18) is more likely to be satisfied. However, Lemma 2 indicates that the task's resource demand under the LO-mode increases when the task's $\mathrm{VD}_{i}$ decreases, which causes Inequation (18) is more likely to be violated. Based on these observations, we propose a greedy virtual deadline assignment algorithm. The major steps of the algorithm are highlighted as follows:

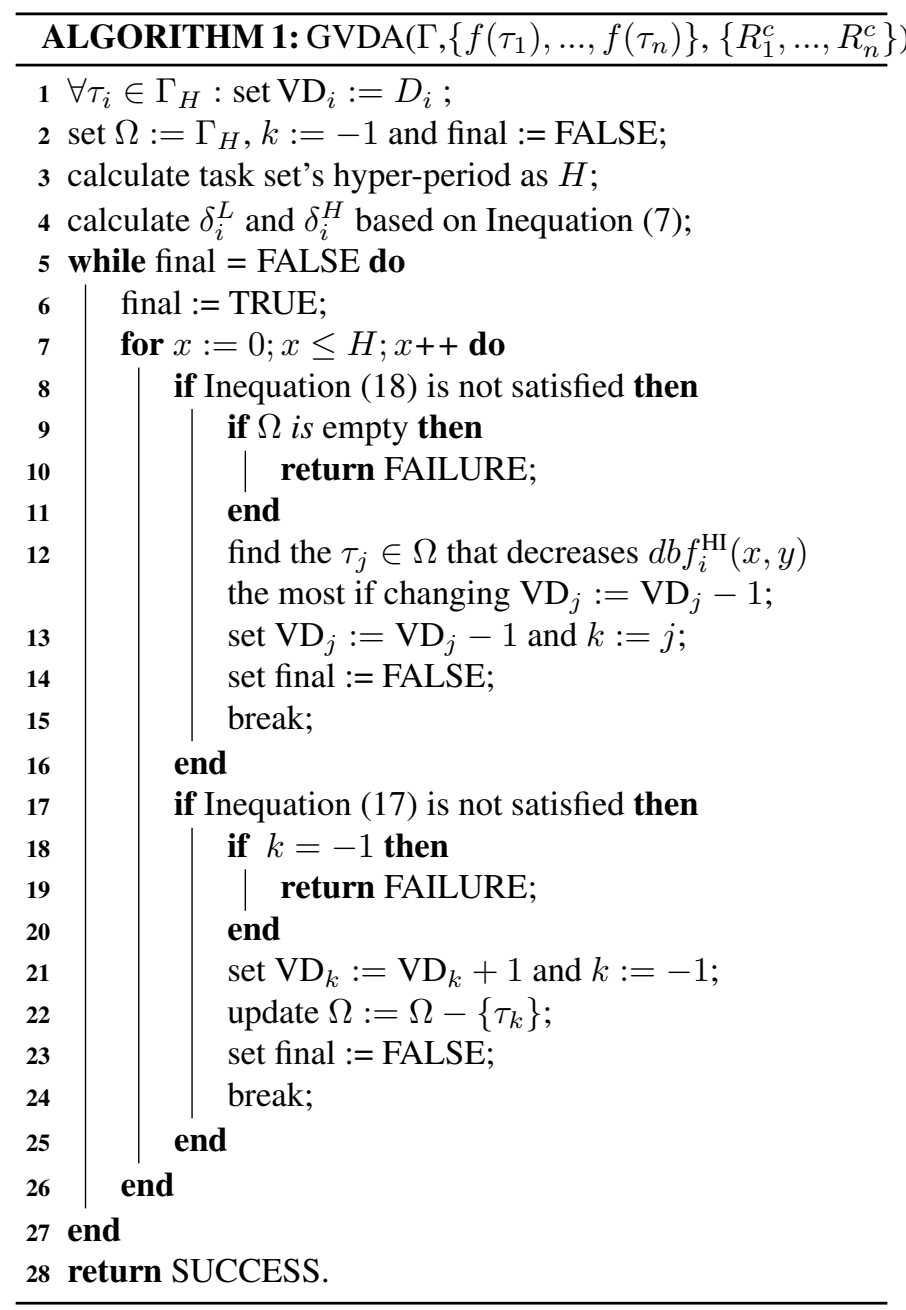

1. Initialization step: we set $x:=0$ and each HI-criticality task $\tau_{i}$ 's virtual deadline $\mathrm{VD}_{i}:=D_{i}$. In addition, we define a new task set which contains tasks whose deadlines 
can be reduced. We call the new task set a reducible task set $\Omega$, and it is initialized to be $\Gamma_{H}$, i.e. $\Omega:=\Gamma_{H}$. In other words, initially every HI-criticality task's deadline is allowed to be reduced.

2. If Inequation (18) is violated: if $\Omega \neq \Phi$, choose a task $\tau \in \Omega$ whose $d b f^{\mathrm{HI}}(x, y)$ decreases the most when its virtual deadline is reduced by 1 time unit and reduce the task's virtual deadline by 1 time unit and reset $\mathrm{x}:=0$; if $\Omega=\Phi$, return FAILURE.

3. If Inequation (17) is violated: undo the last task $\tau_{j}$ 's virtual deadline change, update $\Omega:=\Omega-\left\{\tau_{j}\right\}$ and reset $x:=0$; if there is no change to undo, return FAILURE.

4. Otherwise, increase $x$ by one and repeat step 2)-3) until $x=H$ where $H$ is the hyperperiod of the task set and return SUCCESS.

It is worth pointing out that the algorithm returns SUCCESS only if both Inequation (18) and Inequation (17) are satisfied for every $x$ within range $[0, H]$. The details of our greedy virtual deadline assignment (GVDA) algorithm are given in Algorithm 1. Lines 1-4 are for initialisation, lines 8-16 are the Step 2) and lines 17-25 are the Step 3).

\subsection{Task Execution Frequency Assignment}

For a given frequency to task assignment, the GVDA algorithm can determine if there exists a valid virtual deadline assignment which guarantees both reliability and schedulability constraints. However, there may be multiple task execution frequencies satisfying the schedulability and reliability constraints. Our next step is to decide a valid task execution frequency assignment that minimizes energy consumption of executing the task set.

The proposed heuristic search based approach has the following major steps:

1. Initialization step: all the tasks are assigned $f_{\max }$ as their execution frequencies;

2. Choose one task to scale down its frequency by one level but without violating the reliability and schedulability constraints based on Inequation (8) and (9);

3. Repeat step 2) until no task's execution frequency can be scaled down further.

In step 2), there may be more than one task's processing frequency can be scaled down without violating the reliability and schedulability constraints, which task is chosen to scale down its execution frequency impacts total energy consumption.

Executing a task under a lower frequency reduces energy consumption, but the task takes longer time to complete under a reduced execution frequency. Furthermore, lowering down task's execution frequency increases system transient fault rate which in turn increases task recovery time. We define a metric to measure the energy saving gain over a unit resource demand increase caused by reducing the task's execution frequency from $f\left(\tau_{i}\right)$ to a lower one $f^{\prime}\left(\tau_{i}\right)$, i.e., energy saving efficiency $\operatorname{ESE}\left(\tau_{i}, f\left(\tau_{i}\right), f^{\prime}\left(\tau_{i}\right)\right)$ is defined as

$$
\operatorname{ESE}\left(\tau_{i}, f\left(\tau_{i}\right), f^{\prime}\left(\tau_{i}\right)\right)=\frac{\mathcal{E}\left(f\left(\tau_{i}\right)\right)-\mathcal{E}\left(f^{\prime}\left(\tau_{i}\right)\right)}{\operatorname{Gap}(F)-\operatorname{Gap}\left(F^{\prime}\right)} .
$$

where $F=\left\{f\left(\tau_{1}\right), \ldots, f\left(\tau_{i}\right), \ldots, f\left(\tau_{n}\right)\right\}$ and $F^{\prime}=\left\{f\left(\tau_{1}\right), \ldots, f^{\prime}\left(\tau_{i}\right), \ldots, f\left(\tau_{n}\right)\right\} . \mathcal{E}\left(f\left(\tau_{i}\right)\right)$ is the expected total energy consumption of task $\tau_{i}$ under the assigned frequency $f\left(\tau_{i}\right)$ within one hyper-period $H$, which can be expressed as

$$
\mathcal{E}_{i}\left(f\left(\tau_{i}\right)\right)=\frac{H}{T_{i}} \cdot E_{i}\left(f\left(\tau_{i}\right)\right)
$$

$\operatorname{Gap}(F)$ is defined as

$$
\min _{x \in[0, H]}\left\{x-\sum_{\tau_{i} \in \Gamma} d b f_{i}^{\mathrm{LO}}\left(f\left(\tau_{i}\right), x\right) \mid \sum_{\tau_{i} \in \Gamma} d b f_{i}^{\mathrm{LO}}\left(f\left(\tau_{i}\right), x\right) \neq 0\right\},
$$

where $d b f_{i}^{\mathrm{LO}}\left(f\left(\tau_{i}\right), x\right)$ is the resource demand of task $\tau_{i}$ under frequency $f\left(\tau_{i}\right)$, which can be calculated using 10$]^{1}$

It is worth pointing out that fault recoveries also consume energy, but as the probability of fault occurrence is relatively small, the expected energy consumption for fault recoveries is negligible compared to the energy cost of executing all tasks in the task set.

The function $\operatorname{Gap}(F)$ indicates the gap between the resource demand and resource supply. It can be interpreted as the remaining time to be used for energy saving purposes. Hence, $\operatorname{Gap}(F)-\operatorname{Gap}\left(F^{\prime}\right)$ is the extra execution time demand needed to lower down task $\tau_{i}$ 's processing from $f\left(\tau_{i}\right)$ to $f^{\prime}\left(\tau_{i}\right)$.

With the ESE metric, we given a heuristic search based frequency assignment (HSFA) algorithm that minimizes energy consumption in Algorithm 2

In Algorithm 2, I is an array representing each task's execution frequency. Line 1 initializes each task's working frequency to $f_{\max }$. Function CHECK checks whether task $\tau_{i}$ 's execution frequency can be scaled down by one level without violating the schedulability and reliability constraints. Line 3 finds the task set whose task's execution frequencies can be scaled down. Line 5 selects the one in $\Psi$ that has the largest ESE value.

The pseudo-code code of the function CHECK is given in Algorithm 3. It calls the function GVDA to determine if task $\tau_{i}$ 's execution frequency can be reduced from $I[i]$ to $I[i]+1$.

\section{Evaluation}

In this section, we evaluate the energy saving performance of our proposed approach. In our proposed approach, when the system operate under the HI-mode, we run all the tasks under the maximum frequency $f_{\max }$ and no energy saving can be achieved. Therefore, in the following evaluations, we focus on energy saving comparisons when the system operates under the LO-mode.

${ }^{1} d b f_{i}^{\mathrm{LO}}\left(f\left(\tau_{i}\right), x\right)$ is abbreviated as $d b f_{i}^{\mathrm{LO}}(x)$ in Section 5 to simplify the representation. 

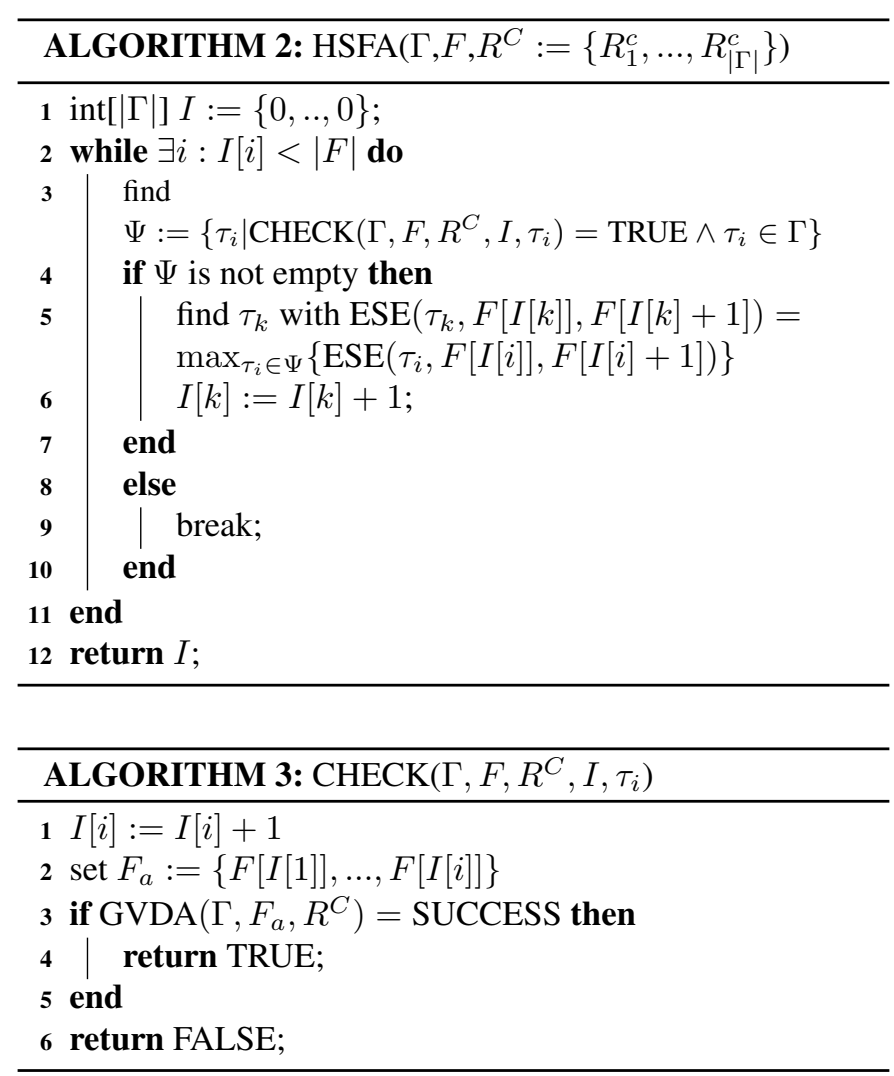

\subsection{Experimental Settings}

The mixed-criticality task sets are generated by UUniForm algorithm [8], which gives an unbiased distribution of task utilization. In particular, the task sets are generated in the following steps:

- use the UUniForm algorithm to generate task utilization, i.e. $u_{H}\left(\tau_{i}\right)$ and $u_{L}\left(\tau_{i}\right)$;

- randomly select task period $T_{i}$ within [20,100];

- set task execution time $C_{i}(H I)$ as $T_{i} \cdot u_{H}\left(\tau_{i}\right)$, where $u_{H}\left(\tau_{i}\right)$ is task $\tau_{i}$ 's HI-mode utilization. For LO-criticality tasks, set $C_{i}(L O)=C_{i}(H I)$; while for HI-criticality tasks, set $C_{i}(L O)=\mu \cdot C_{i}(H I)$, where $\mu$ is a random value within the range of $[0.3,0.5]$.

For energy model parameters, we assume $P_{\text {ind }}=0.1, C_{\text {ef }}=$ 1 , and $\theta=3$. The available frequencies are set as $F=\{0.4,0.6,0.8,1\}$. For transient fault model parameters, we set $\lambda_{0}=10^{-6}$ and $d=3$.

We evaluate our proposed approach by comparing it with the two heuristics, i.e. the smallest utilization first (SUF) approach and the heuristic search based energy minimization (HSEM) approach presented in our earlier work [17] from the following perspectives:

- sensitivity to LO-mode and HI-mode utilization;

- sensitivity to the number of tasks in the task set;

- sensitivity to the fault arrival rate.

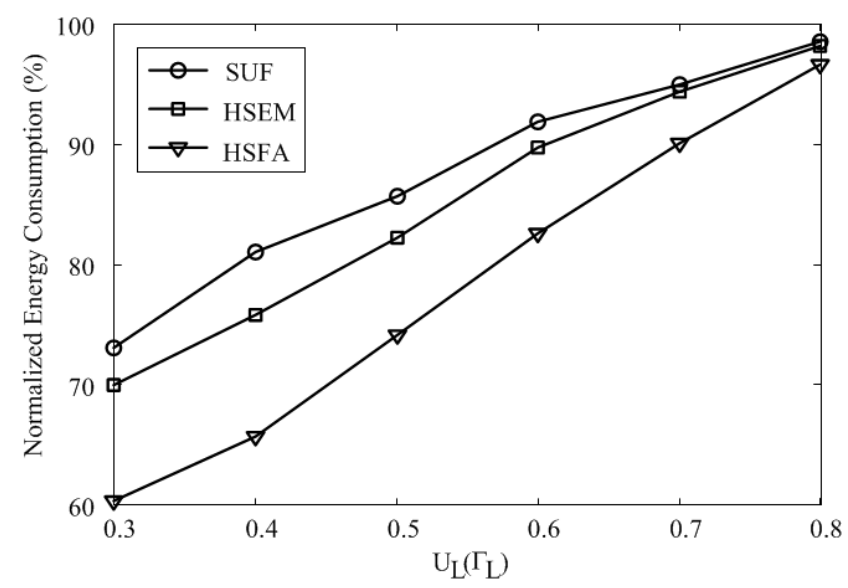

Figure 4: Energy consumption under varied $U_{L}\left(\Gamma_{L}\right)$

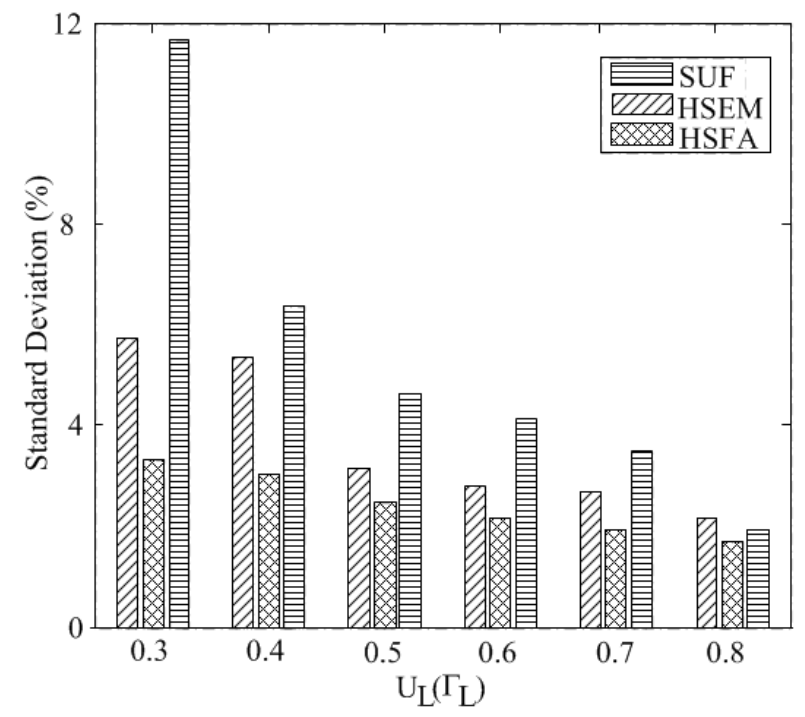

Figure 5: Energy consumption variation under varied $U_{L}\left(\Gamma_{L}\right)$

Though Huang et al.'s approach [15] does not guarantee the reliability constraint and hence can not be used to solve our formulated problem, we still add the comparison between our HSFA approach and Huang et al.'s approach as an additional set of experiments.

\subsection{Experiment Results and Discussions}

For comparison purposes, energy consumption is normalized to the value when all tasks run under the highest frequency $f_{\max }=1$. For each experiment, we run the compared algorithms for 100 randomly generated task sets, the average energy consumption and the standard derivation are used in the comparisons.

\subsubsection{Sensitivity to LO-mode and HI-mode utilization}

In the first set of experiments, each task set has six tasks. Among which, three are of HI-criticality and the other three are of LO-criticality. We set $U_{H}\left(\Gamma_{H}\right)=0.3$ and vary $U_{L}\left(\Gamma_{L}\right)$ 


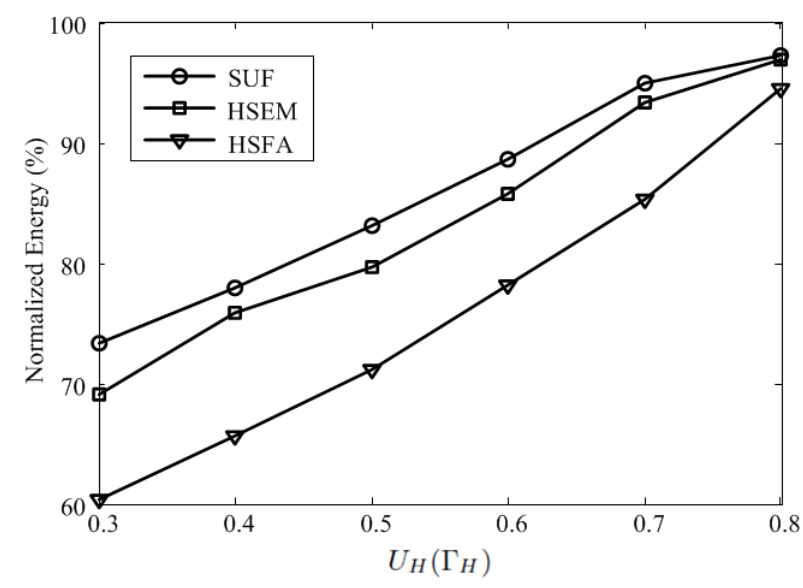

Figure 6: Energy consumption under varied $U_{H}\left(\Gamma_{H}\right)$

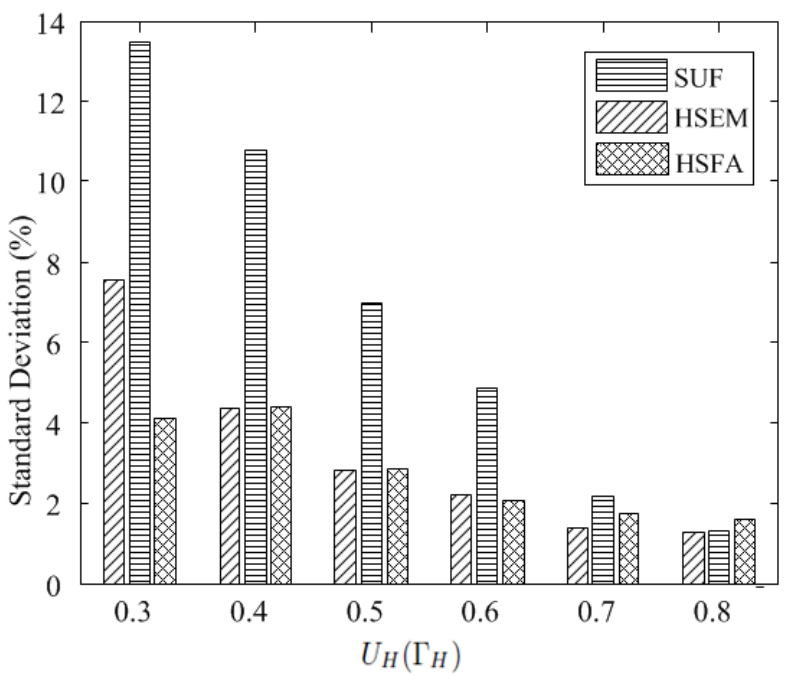

Figure 7: Energy consumption variation under varied $U_{H}\left(\Gamma_{H}\right)$

from 0.3 to 0.8 with step of 0.1 . The average normalized energy consumption is illustrated in Figure 4 It is not hard to find that all compared approaches consume more energy under higher task set utilization. This is because that task set of higher utilization has less slack time, hence, less chance to scale down task working frequencies for energy saving purposes. In addition, with the SUF and HSEM approaches, each HI-criticality task is assumed to have a carry-over instance and hence the resource demand calculation is too pessimistic. With the HSFA algorithm, the over demand is excluded and the energy saving performance is improved. These explain why the HSFA approach can save up to $15 \%$ and $10 \%$ more energy than SUF and HSEM approaches, respectively.

As shown in Figure 5 the standard derivation of all the compared approaches become smaller when $U_{L}\left(\Gamma_{L}\right)$ is increased. Under lower $U_{L}\left(\Gamma_{L}\right)$, the SUF algorithm has much higher variation than the HSEM and the HSFA algorithm. However, when the $U_{L}\left(\Gamma_{L}\right)$ increases, the variation of the three approaches almost converge at the same point.

For the second set of experiments, we fix $U_{L}\left(\Gamma_{L}\right)=0.3$

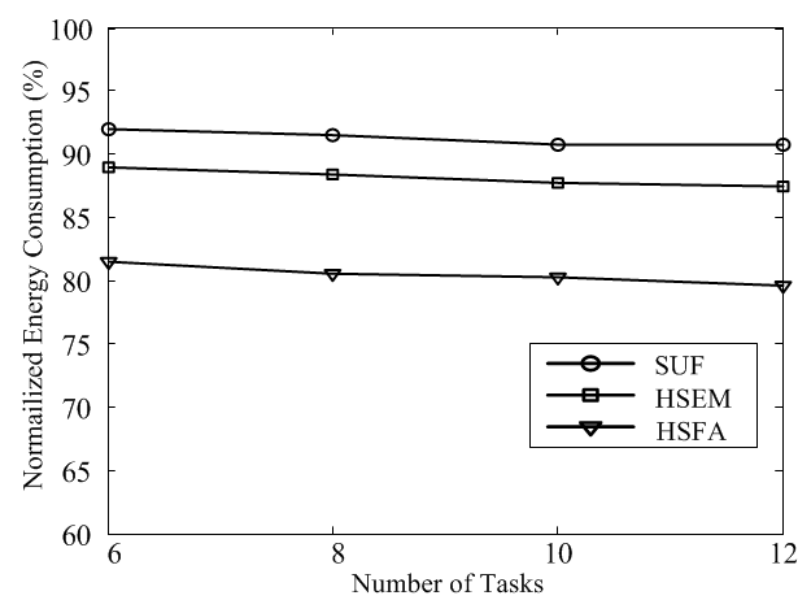

Figure 8: Energy consumption under different number of tasks

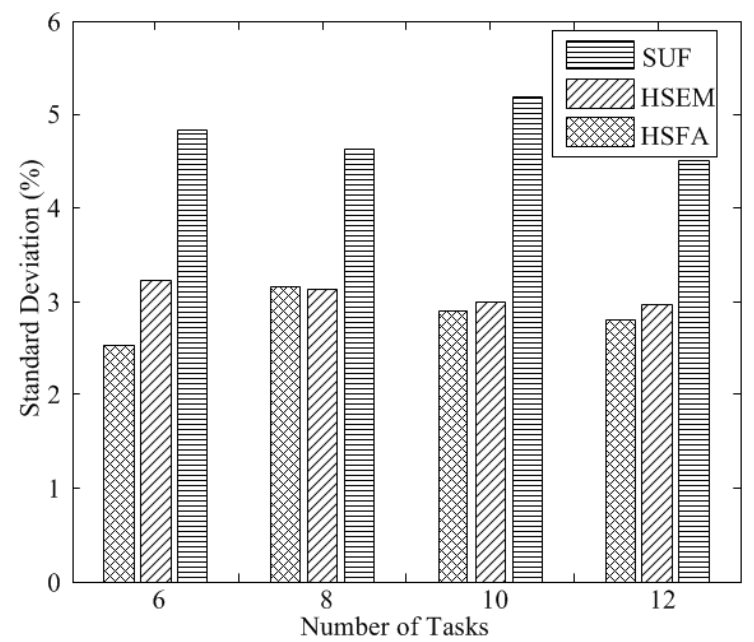

Figure 9: Energy consumption variation under different number of tasks

and vary $U_{H}\left(\Gamma_{H}\right)$ from 0.3 to 0.8 . The experimental results are depicted in Figure 6 and Figure 7 It is not hard to find that HI-mode utilization also impacts the system's energy saving under the LO-mode. The reason is as follows. The system initially runs under the LO-mode and may change to the HImode at any time point. In order to guarantee $\mathrm{HI}$-criticality task deadlines under the HI-mode, enough slack time must be reserved under the LO-mode to accommodate the possible task overruns. Higher HI-mode utilization means more slack time reservation, and hence, as shown in Figure 6 , less energy saving can be achieved.

\subsubsection{Sensitivity to the number of tasks in the task set}

In this set of experiments, we set $U_{H}\left(\Gamma_{H}\right)=0.3, U_{L}\left(\Gamma_{L}\right)=$ 0.6 and increase the number of tasks from 6 to 12 (same number of LO-criticality and HI-criticality tasks) to evaluate how the number of tasks impacts the proposed approach. The experimental results depicted in Figure 8 and Figure 9 reveal that the energy saving performance is slightly improved with the increase of the number of tasks in the task set. 


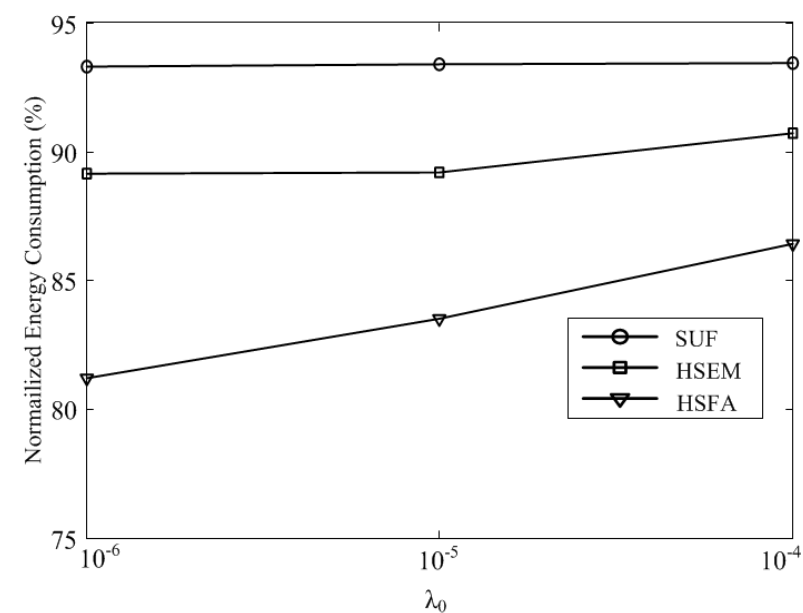

Figure 10: Energy consumption under different $\lambda_{0}$

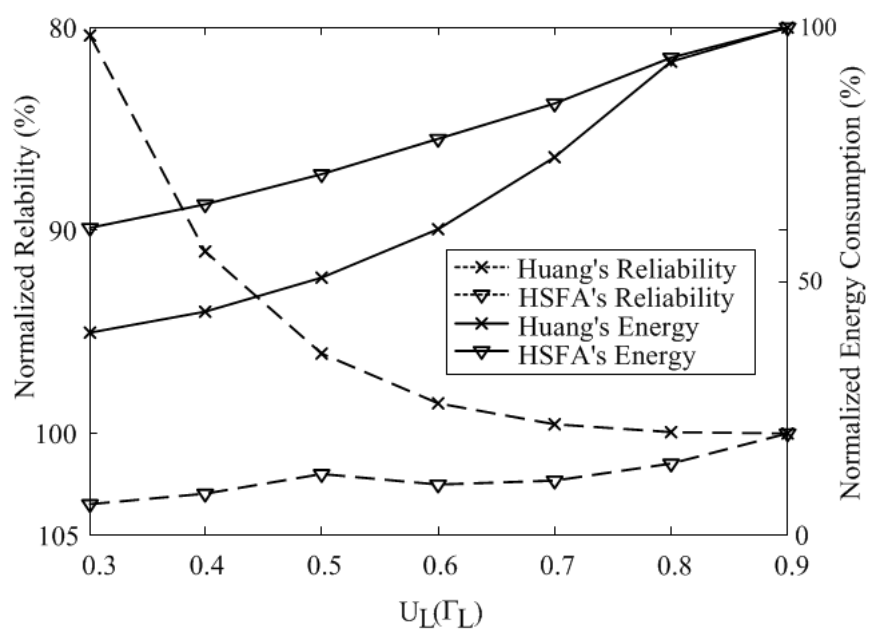

Figure 11: Comparisons between HSFA and Huang's Approach

By comparing the Figure 6 and Figure 4 we can conclude that the number of tasks has less impact on the energy saving performance than task utilization.

\subsubsection{Sensitivity to the fault arrival rate}

In this subsection, we evaluate the impact of fault arrival rate by varying $\lambda_{0}$ from $10^{-4}$ to $10^{-6} . U_{H}\left(\Gamma_{H}\right), U_{L}\left(\Gamma_{L}\right)$ and the number of tasks are set to $0.3,0.6$ and 6 , respectively. As illustrated in Figure 10 , the increase of fault arrival rate $\lambda_{0}$ will degrade the energy saving performance. Larger $\lambda_{0}$ indicates more slack time to be reserved for fault recovery, and hence, less slack time remains for energy saving purpose.

\subsubsection{Comparison with Huang et al.'s approach}

We also set up experiments to compare Huang et al.'s approach [15] with our HSFA approach. We set $U_{H}\left(\Gamma_{H}\right)=0.3$ and increase $U_{L}\left(\Gamma_{L}\right)$ from 0.3 to 0.8 . According to the results illustrated in Figure 11. Huang et al.'s approach has better energy saving performance when $U_{L}\left(\Gamma_{L}\right)$ is less than 0.9 . When the $U_{L}\left(\Gamma_{L}\right)$ is set at 0.9 , both HSFA and Huang et al.'s ap- proaches converge to the same point and almost no energy saving can be achieved. Though Huang et al.'s approach can save more energy under low utilization, but their solution can not meet the reliability constraint. As shown in Figure 11, when $U_{L}\left(\Gamma_{L}\right)=0.3$, the achieved reliability is only $80 \%$ of the requirement. Hence, Huang et al.'s approach can not be used to solve our formulated problem.

\section{Conclusion}

In this paper, we have studied the mixed-criticality task set scheduling problem with the goal of minimizing system energy consumption while at the same time guaranteeing the satisfaction of task set deadline and reliability requirements. We have first established the theoretical foundation that decides under given task virtual deadline and execution frequency assignments whether the tasks' deadline and reliability constraints can be satisfied under EDF-VD scheduling algorithm. Based on the theoretic analysis, we have developed a heuristic search based frequency assignment algorithm that minimizes system's energy consumption and at the same time guarantees both task reliability and schedulability constraints are satisfied. Empirical evaluations show that the proposed approach can save up to $10 \%$ more energy compared to our earlier work in the literature.

\section{Acknowledgement}

This work was supported, in part, by NSF CNS 1018731 and NSF Career 0746643.

\section{References}

[1] Axer, P., Sebastian, M., Ernst, R., 2011. Reliability analysis for mpsocs with mixed-critical, hard real-time constraints. In: Proceedings of the Seventh IEEE/ACM/IFIP International Conference on Hardware/Software Codesign and System Synthesis. CODES+ISSS '11. ACM, New York, NY, USA, pp. 149-158.

[2] Aydin, H., Devadas, V., Zhu, D., dec. 2006. System-level energy management for periodic real-time tasks. In: Proceedings of 27 th IEEE International Real-Time Systems Symposium. RTSS. pp. 313 -322.

[3] Aydin, H., Zhu, D., oct. 2009. Reliability-aware energy management for periodic real-time tasks. Computers, IEEE Transactions on 58 (10), 1382 $-1397$.

[4] Barhorst, J., Belote, T., Binns, P., Hoffman, J., Paunicka, J., Sarathy, P., Scoredos, J., Stanfill, P., Stuart, D., Urzi, R., Apr. 2009. A research agenda for mixed-criticality systems. In: Cyber-Physical Systems Week.

[5] Baruah, S., Bonifaci, V., D’Angelo, G., Li, H., Marchetti-Spaccamela, A., Megow, N., Stougie, L., Aug 2012. Scheduling real-time mixed-criticality jobs. Computers, IEEE Transactions on 61 (8), 1140-1152.

[6] Baruah, S., Bonifaci, V., D’Angelo, G., Li, H., Marchetti-Spaccamela, A., Van der Ster, S., Stougie, L., July 2012. The preemptive uniprocessor scheduling of mixed-criticality implicit-deadline sporadic task systems. In: Real-Time Systems (ECRTS), 2012 24th Euromicro Conference on. pp. 145-154.

[7] Baruah, S., Vestal, S., July 2008. Schedulability analysis of sporadic tasks with multiple criticality specifications. In: Real-Time Systems, 2008. ECRTS '08. Euromicro Conference on. pp. 147-155.

[8] Bini, E., Buttazzo, G., 2005. Measuring the performance of schedulability tests. Real-Time Systems 30 (1-2), 129-154.

[9] Burd, T. D., Brodersen, R. W., 1995. Energy efficient cmos microprocessor design. In: Proceedings of the 28th Hawaii International Conference on System Sciences. HICSS. pp. 288-297. 
[10] Burns, A., Davis, R. I., February 2013. Mixed criticality systems: A review. Tech. Rep. MCC-1(b), Department of Computer Science, University of York, East Lansing, Michigan.

[11] Buttazzo, G., Lipari, G., Abeni, L., Dec 1998. Elastic task model for adaptive rate control. In: Real-Time Systems Symposium, 1998. Proceedings., The 19th IEEE. pp. 286-295.

[12] Ekberg, P., Yi, W., July 2012. Bounding and shaping the demand of mixed-criticality sporadic tasks. In: Real-Time Systems (ECRTS), 2012 24th Euromicro Conference on. pp. 135-144.

[13] Ekberg, P., Yi, W., 2014. Bounding and shaping the demand of generalized mixed-criticality sporadic task systems. Real-Time Systems 50 (1), 48-86.

[14] Guo, Y., Zhu, D., Aydin, H., Aug 2013. Generalized standby-sparing techniques for energy-efficient fault tolerance in multiprocessor real-time systems. In: Embedded and Real-Time Computing Systems and Applications (RTCSA), 2013 IEEE 19th International Conference on. pp. 62-71.

[15] Huang, P., Kumar, P., Giannopoulou, G., Thiele, L., 2014. Energy efficient dvfs scheduling for mixed-criticality systems. In: Proceedings of the 14th International Conference on Embedded Software. EMSOFT '14. pp. 11:1-11:10.

[16] Koren, G., Shasha, D., Dec 1995. Skip-over: algorithms and complexity for overloaded systems that allow skips. In: Real-Time Systems Symposium, 1995. Proceedings., 16th IEEE. pp. 110-117.

[17] Li, Z., Hua, X., Guo, C., Ren, S., Nov 2014. Empirical study of energy minimization issues for mixed-criticality systems with reliability constraint. In: Low-Power Dependable Computing (LPDC), 2014 1st Workshop on.

[18] Li, Z., Wang, L., Ren, S., Quan, G., June 2013. Energy minimization for checkpointing-based approach to guaranteeing real-time systems reliability. In: Object/Component/Service-Oriented Real-Time Distributed Computing (ISORC), 2013 IEEE 16th International Symposium on. pp. $1-8$.

[19] Niu, L., Xu, J., 2015. Improving schedulability and energy efficiency for window-constrained real-time systems with reliability requirement. Journal of Systems Architecture 61 (5C6), 210 - 226.

[20] Park, T., Kim, S., 2011. Dynamic scheduling algorithm and its schedulability analysis for certifiable dual-criticality systems. In: Proceedings of the Ninth ACM International Conference on Embedded Software. EMSOFT. ACM, New York, NY, USA, pp. 253-262.

[21] Pathan, R., 2014. Fault-tolerant and real-time scheduling for mixedcriticality systems. Real-Time Systems 50 (4).

[22] Su, H., Zhu, D., March 2013. An elastic mixed-criticality task model and its scheduling algorithm. In: Design, Automation Test in Europe Conference Exhibition (DATE), 2013. pp. 147-152.

[23] Zhang, T., Guan, N., Deng, Q., Yi, W., June 2014. On the analysis of edf-vd scheduled mixed-criticality real-time systems. In: Industrial Embedded Systems (SIES), 2014 9th IEEE International Symposium on. pp. 179-188.

[24] Zhao, B., Aydin, H., Zhu, D., 2009. Enhanced reliability-aware power management through shared recovery technique. In: Proceedings of the International Conference on Computer-Aided Design. ICCAD. pp. 6370 .

[25] Zhao, B., Aydin, H., Zhu, D., 2011. Generalized reliability-oriented energy management for real-time embedded applications. In: Proceedings of the Design Automation Conference. DAC. pp. 381-386.

[26] Zhao, B., Aydin, H., Zhu, D., 2012. Energy management under general task-level reliability constraints. 2013 IEEE 19th Real-Time and Embedded Technology and Applications Symposium (RTAS) 0, 285-294.

[27] Zhu, D., 2011. Reliability-aware dynamic energy management in dependable embedded real-time systems. ACM Trans. Embed. Comput. Syst. $10(2), 1-27$.

[28] Zhu, D., Melhem, R., Mosse, D., 2004. The effects of energy management on reliability in real-time embedded systems. In: Proceedings of the IEEE/ACM International conference on Computer-aided design. ICCAD. pp. $35-40$. 